\title{
Optical Diagnostics for Plasma Wakefield Accelerators
}

\author{
E. Oz ${ }^{1}$, C.D. Barnes ${ }^{2}$, C.E. Clayton ${ }^{3}$, F.J. Decker ${ }^{2}$, S. Deng ${ }^{1}$, M.J. Hogan ${ }^{2}$, \\ C. Huang ${ }^{3}$, R. Iverson ${ }^{2}$, D.K. Johnson ${ }^{3}$, C. Joshi ${ }^{3}$, T. Katsouleas ${ }^{1}$, P. \\ $\mathrm{Krejcik}^{2}$, W. Lu ${ }^{3}$, K.A. Marsh ${ }^{3}$, P. Muggli ${ }^{1}$, C. O’Connell ${ }^{2}$, D. Walz \\ ${ }^{1}$ University of Southern California 90089 \\ ${ }^{2}$ Stanford Linear Accelerator Center 94025 \\ ${ }^{3}$ University of California, Los Angeles 90095
}

\begin{abstract}
The amount of spectral energy released by the plasma as visible light is proportional to the amount of energy loss of the beam, a simple beam independent diagnostic which is an ideal tool to help understand wake field plasma interaction is presented here. Continuum light emitted by particles through Cerenkov and/or optical transition processes also indicate the appearance of plasma particles trapped by the large amplitude plasma wake of the PWFA.
\end{abstract}

Plasma accelerators have made tremendous progress in the last year(see for example these Proceedings).Energy gains greater than $3 \mathrm{GeV}$ were measured in an electron beam-driven plasma wakefield accelerator (PWFA) experiment using the ultrarelativistic $(28.5 \mathrm{GeV})$, ultra-short $(<100 \mathrm{fs})$, high-current $(>10 \mathrm{kA})$ bunches now available at the Stanford Linear Accelerator Center (SLAC). An imaging magnetic spectrometer was developed to un-ambiguously measure the beam energy spectrum after it interacted with the plasma, with an accuracy of the order of $40 \mathrm{MeV}$. The spectrometer is composed of six quadrupole and six dipole magnets and images the beam at the plasma entrance or exit onto a thin piece of aerogel located $\approx 25 \mathrm{~m}$ downstream from the plasma. The Cherenkov light emitted by the beam when traversing the $1 \mathrm{~mm}$ thick piece of aerogel is imaged onto a CCD camera. Detailed energy spectra are obtained from the careful analysis of the images of the beam dispersed in energy. In previous experiments with longer bunches $(\approx 2.2 \mathrm{ps})$, the Cherenkov light was dispersed in time with an $\approx 1 \mathrm{ps}$ resolution to temporally resolve both the energy loss and gain imparted to the electron [1] and positron [2] bunches by the PWFA action. However, this analysis procedure is relatively complicated and time consuming and cannot be performed on a bunch-to-bunch basis. Since a fraction of the beam energy is deposited in the plasma, analyzing the light emitted by the plasma could provide important and immediate information about the PWFA interaction. In this paper we report on the plasma light measurements performed during the E-164-X PWFA experiment. We show that the amount of light emitted by the neutral lithium atoms correlates with the mean beam energy loss and can be used as a simple and on- 
line measurement. Analysis of the plasma light also reveals the presence of plasma trapped particles, and could also yield information about the various ionization processes occurring in the experiment.

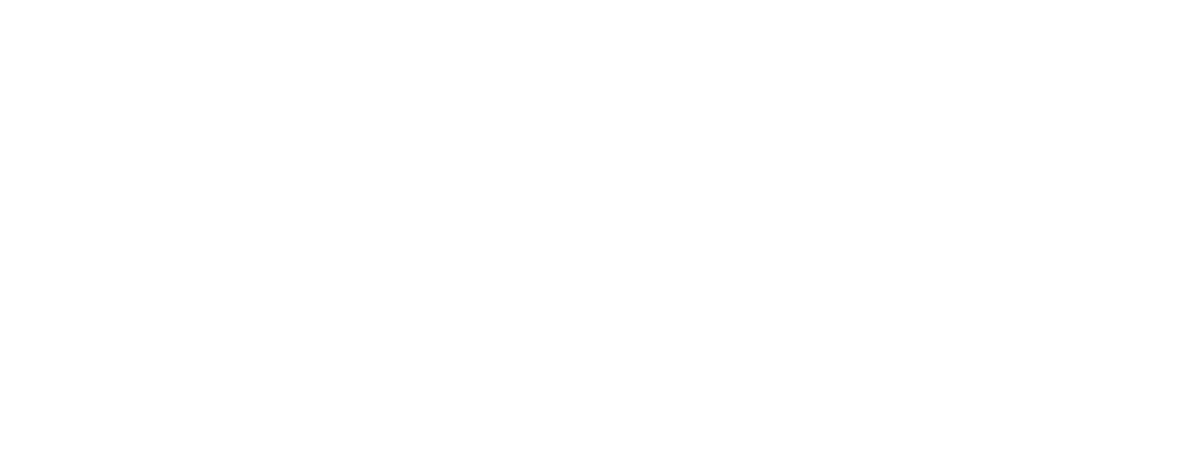

FIGURE 1: Experimental Setup (not to scale).

The PWFA is an energy transformer in which energy is extracted from the bunch core particles (or from a driver bunch) and is transferred to the plasma electrons in the form of a large amplitude plasma wave or wake. In single bunch experiments, such as the E-164X experiment at SLAC, particles in the back of the same bunch can absorb some fraction of the wake energy and therefore be accelerated. In two-bunch experiments, the process can be made more efficient by placing a second bunch of lower charge after the driving, high-charge bunch and thereby loading the wake. In single bunch experiments the amount of energy extracted for the plasma wake is small when compared to the amount deposited in the plasma, and most of the wake energy is dissipated in the plasma. The amount of energy deposited in the plasma can be evaluated by measuring the average bunch energy after interaction with the plasma. In the case of the SLAC experiment, with a typical average energy loss of $\approx 1 \mathrm{GeV}$ for the $\approx 3 \mathrm{nC}$ bunch, the energy deposited in the plasma is of the order of $3 \mathrm{~J}$. Note that a small fraction of the energy lost by the bunch particles is emitted as forward-going $\mathrm{x}$ rays, a radiation process resulting for the bunch particles betatron motion along the plasma $[3,4]$. The wake energy is deposited in the plasma which has a length equal to the lithium column length, $\approx 10 \mathrm{~cm}$, and a radius given by the ionization radius of $\approx 150 \mu \mathrm{m}$, for the $12 \mu \mathrm{m}$ radius electron bunch case [5]. With a typical plasma density of $2.5 \times 10^{17} \mathrm{~cm}^{-3}$, the average energy gained by each plasma electron is of the order of $10 \mathrm{keV}$. In the experiment, the plasma is created through field ionization a lithium vapor by the large bunch radial electric field. A fraction of the beam energy is therefore used to ionize the lithium vapor, which has an ionization potential of $5.39 \mathrm{eV}$. However, with the above mentioned density and volume, the energy loss to ionization is of the order of $1.5 \mathrm{~mJ}$, and is very small when compared to the wake energy. The energy gained by the plasma electrons can be dissipated as bremsstrahlung continuum radiation through collision with the plasma ions, or through collision and excitation of the various atomic and ionic species present in the plasma. The excited atoms and ions later release their energy through atomic radiation at 
discrete frequencies. Analyzing the light emitted by the plasma can therefore yield information about the various ionization levels present in the plasma, as well as about the strength of the interaction between the particle beam and the plasma.

A schematic of the experiment is shown on Fig. 1. After interacting with plasma, the beam traverses a $1 \mu \mathrm{m}$ thick titanium foil placed at $45^{\circ}$ relative to the beam propagation direction. This foil is used to extract the light emitted by the plasma from the beam line, and to shield the second foil from this light. The second foil is used to monitor the transverse beam properties using optical transition radiation (OTR). The plasma light is then directed toward and focused onto the slit of an $27 \mathrm{~cm}$ focal length imaging optical spectrograph. A gated intensified camera with $1024 \times 256,27 \mu \mathrm{m}$ square pixel is placed in the image plane of the spectrograph. The wavelength range is $650 \mathrm{~nm}$, and the resolution about $2.5 \mathrm{~nm}$ with a $150 \mathrm{gr} / \mathrm{mm}$ grating blazed at $560 \mathrm{~nm}$, and $77 \mathrm{~nm}$, and the resolution about $0.3 \mathrm{~nm}$ with a $1200 \mathrm{gr} / \mathrm{mm}$ grating blazed at $500 \mathrm{~nm}$. The low dispersion grating is used to monitor the entire visible spectrum, while the higher dispersion grating is used to monitor the width of individual atomic/ionic lines. Figure 2 shows a typical plasma light image dispersed in wavelength, as well as the corresponding energy spectrum obtained by summing the image along its vertical dimension. The plasma light spectrum is composed of atomic lines from the different atoms present in the heat-pipe oven ( $\mathrm{Li}, \mathrm{He}, \mathrm{Fe})$.

In the experiment the relative bunch length and bunch current distribution vary form bunch to bunch. The incoming bunch energy spectrum and the total coherent transition radiation (CTR) energy emitted by the bunch when traversing a foil located upstream from the plasma are monitored on a bunch-to-bunch basis. The CTR energy is expected to be inversely proportional to the bunch length and is used as a relative bunch length monitor. The incoming bunch energy spectrum will be used to retrieve the bunch current distribution for a detailed analysis of the bunch energy gain and loss. The event of Fig. 2 corresponded to a low CTR energy value, and therefore to a relatively long bunch length. In contrast, Fig. 3 shows the plasma light image and spectrum obtained with a shorter bunch, as indicated by the corresponding higher CTR energy. A continuous component to the spectrum is visible on Fig. 3. Preliminary analysis indicates that the continuum component of the spectrum is the result of plasma electrons trapped by the plasma wake, and exiting the plasma together with the incoming electron bunch. These particles can emit Cherenkov radiation in the He buffer gas located between the plasma and the plasma light extraction foil, and OTR emitted when they traverse the plasma light extraction foil. The trapped particles can be monitored by measuring the amount of light they emit, for example by calculating the relative light intensity recorded in a wavelength range on Figs 2 and 3 where there is no atomic line radiation. Figure 4 shows the relative amount of light recorded between 515 and $528 \mathrm{~nm}$ as a function of the CTR energy. Figure 4a shows a clear threshold at a relative CTR energy of $\approx 550$. Above this value the amount of light becomes much larger, and has rather large amplitude variations. A charge monitor (toroid) located immediately after the plasma light extraction foil also indicates an excess of charge above the relative CTR energy value of 550 (Fig. 4b). The origin and the characteristics of the trapped particles will be described elsewhere. 

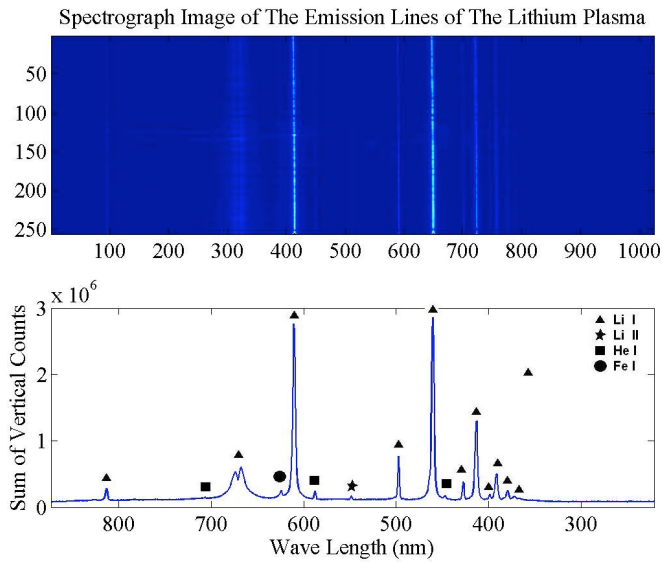

FIGURE 2. Typical plasma light image and its corresponding spectrum obtained with the $150 \mathrm{gr} / \mathrm{mm}$ grating. Atomic lines from LiI,, LiII, Fe and He are visible. The Fe lines probably originate from iron emitted by the Li heat-pipe oven maintained at a temperature $>900^{\circ} \mathrm{C}$. The He lines originate from the excitation of the heat-pipe buffer gas, He at room temperature.
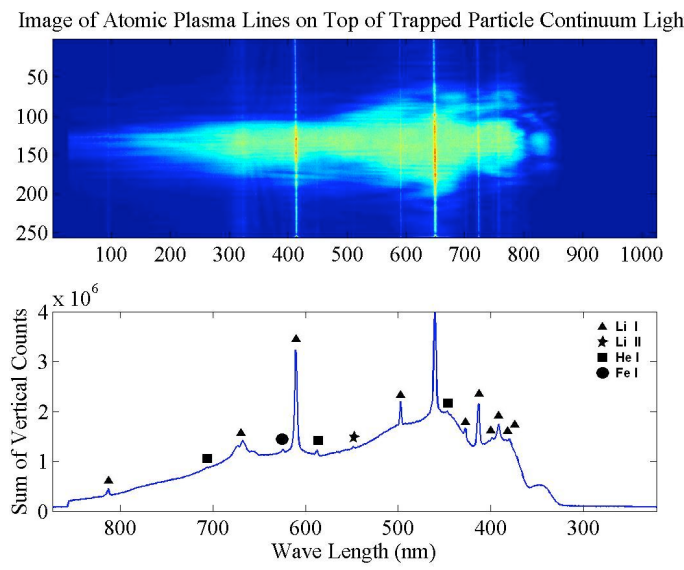

FIGURE 3. Plasma light image and corresponding spectrum, obtained with shorter bunch as that of Fig.2, as indicated by the higher CTR energy. 

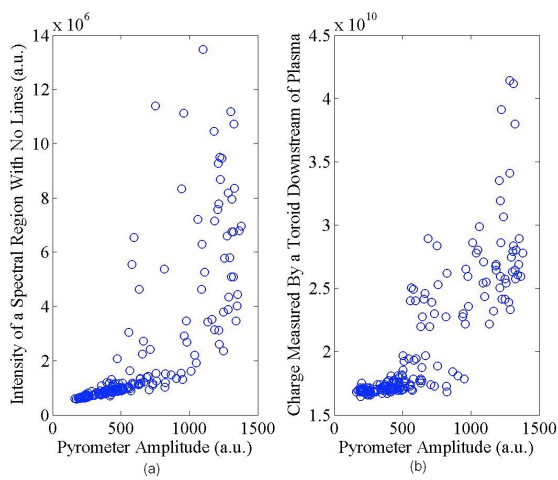

FIGURE 4 (a) the relative amount of light recorded between 515 and $528 \mathrm{~nm}$ (b) Amount of Charge recorded by a Toroid (Charge Monitor Device), versus the CTR energy.

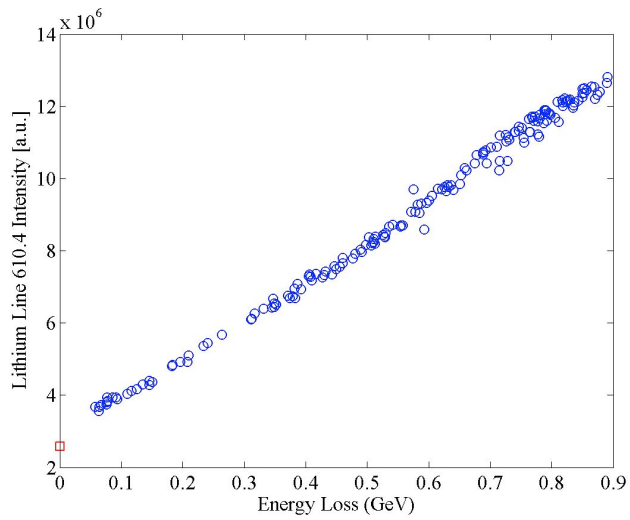

FIGURE 5. Intensity of the lithium atomic line at $610 \mathrm{~nm}$ as a function of the mean beam energy as recorded after the plasma by a beam position monitor located $\approx 30 \mathrm{~cm}$ downstream from the aerogel (see Fig. 1), i.e., $30 \mathrm{~cm}$ downstream from the magnetic spectrometer image plane. The plasma density is constant at $1 \times 10^{17} \mathrm{~cm}^{-3}$ (blue circles), the red square corresponds to plasma off events, and the energy loss varies because the bunch length varies from bunch to bunch. The line intensity is proportional to the mean beam energy, i.e., to the beam energy lost to the plasma wake. 
In the experiment the energy spectrum of the bunch after the plasma is obtained by recoding the Cherenkov light emitted by the beam when traversing a thin piece of aerogel placed in the image plane of the magnetic spectrometer (see Fig. 1). However, the analysis of the beam images is time consuming and cannot be performed at $1 \mathrm{~Hz}$, the beam repetition rate during the experiment. The beam position along the beam line is monitored using beam position monitors (BPMs). In particular, a BPM located about $30 \mathrm{~cm}$ downstream from the aerogel can be used to measure the mean beam energy by measuring the mean beam position in the vertical dispersive plane of the magnetic spectrometer. We refer to this BPM as the energy BPM. However, the range over which the BPM reading remains linear with the beam position is limited and the BPM measurement is also severely perturbed when beam particles are intercepted by the BPM's electrodes, which happens when both the beam energy gain and loss are large. The BPM is also outside of the imaging spectrometer image plane, and is therefore somewhat sensitive to the presence of a beam tail that can contribute to and perturb the beam energy measurement.

The intensity of the plasma light spectral lines can be obtained from the dispersed images acquired for each bunch (see Figs. 2 and 3), or can be obtained using a photodiode placed after the spectrograph and recording the relative intensity of a single line. Figure 5 shows the intensity of one of the strongest LiI lines, at $610 \mathrm{~nm}$, as a function of the mean beam energy as measured by the energy BPM. The linear relationship between these two quantities indicates that the beam energy loss can be monitored on a shot to shot basis simply by recording the amount of light emitted in a particular atomic line of the plasma atoms, a measurement completely independent form the quality of the beam exiting the plasma. This measurement does not suffer from the BPM measurements limitations and can be used over a very large range of energy loss. Note that similar linear relationships are also obtained from other LiI lines.

Additional information about the plasma can be obtained by gating the plasma light in time. Gating the plasma light shows that the atomic emission process lasts for more than $100 \mu \mathrm{s}$. The typical life time of the LiI excited levels is of the order of a few hundreds of nanoseconds, and the emission of plasma light many microseconds after the beam has left the plasma could indicate that both wake fields and plasma density can last for microseconds. This could have important consequences for a future linear collider afterburner in which the individual bunches in the bunch train are separated by only a few to a few hundreds of nanoseconds [6]. Gating the plasma light also confirmed that the continuum emission was prompt with the beam passage. Continuum light emission is only observed during the shortest gate time ( $3 \mathrm{~ns}$ ) around the beam passage time. Atomic spectral line emission was observed to start only approximately $40 \mathrm{~ns}$ later.

\section{CONCLUSIONS}

Monitoring the light emitted by the plasma in PWFA experiments can provide valuable and independent information about the beam/plasma interaction. In particular, in the present experiment, the atomic line intensity of the neutral gas/vapor 
is proportional to the beam energy loss. Appearance of atomic lines can also be used to monitor the various ionization levels existing in the plasma. Recording the light emitted through Cherenkov and OTR processes also provides valuable information about the appearance of trapped particles in the PWFA.

\section{ACKNOWLEDGEMENTS}

This work is supported by the US Department of Energy. The aerogel is supplied by Dr. P. Tsou from JPL.

\section{REFERENCES}

1. P. Muggli et al., Phys. Rev. Lett. 93 , 014802 (2004).

2. B.E. Blue et al., Phys. Rev. Lett. 90, 214801 (2003).

3. S. Wang et al., Phys. Rev. Lett. 88, 135004 (2002).

4. D. Johnson et al, theses Proceedings.

5. P. Muggli, E. Oz, and K.A. Marsh, AIP Conference Proceedings 647, New York: American Institute of Physics, 620 (2002).

6. P. Muggli and J.S.T. Ng, these Proceedings. 